\title{
ROS-Dependent Neuroprotective Effects of NaHS in Ischemia Brain Injury Involves the PARP/AIF Pathway
}

\author{
Qian Yua Zhihong Lu ${ }^{\mathrm{b}}$ Lei Tao ${ }^{\mathrm{a}}$ Lu Yang $^{\mathrm{a}}$ Yu Guo ${ }^{\mathrm{a}}$ Yonghui Yang ${ }^{\mathrm{a}} \quad$ Xude Sun ${ }^{\mathrm{a}}$ \\ Qian Dinga \\ aDepartment of Anesthesiology, Tangdu Hospital, Fourth Military Medical University, Xi'an,

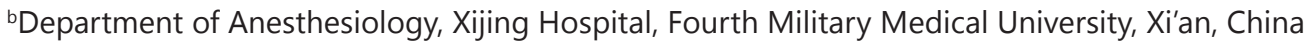

\section{Key Words}

$\mathrm{NaHS} \cdot$ Ischemia-Reperfusion $\bullet$ ROS $•$ Apoptosis $・$ Neuroprotective

\begin{abstract}
Background/Aims: Stroke is among the top causes of death worldwide. Neuroprotective agents are thus considered as potentially powerful treatment of stroke. Methods: Using both HT22 cells and male Sprague-Dawley rats as in vitro and in vivo models, we investigated the effect of $\mathrm{NaHS}$, an exogenous donor of $\mathrm{H}_{2} \mathrm{~S}$, on the focal cerebral ischemia-reperfusion (I/R) induced brain injury. Results: Administration of NaHS significantly decreased the brain infarcted area as compared to the I/R group in a dose-dependent manner. Mechanistic studies demonstrated that NaHS-treated rats displayed significant reduction of malondialdehyde content, and strikingly increased activity of superoxide dismutases and glutathione peroxidase in the brain tissues compared with I/R group. The enhanced antioxidant capacity as well as restored mitochondrial function are NaHS-treatment correlated with decreased cellular reactive oxygen species level and compromised apoptosis in vitro or in vivo in the presence of NaHS compared with control. Further analysis revealed that the inhibition of PARP-1 cleavage and AIF translocation are involved in the neuroprotective effects of NaHS. Conclusion: Collectively, our results suggest that NaHS has potent protective effects against the brain injury induced by I/R. NaHS is possibly effective through inhibition of oxidative stress and apoptosis.
\end{abstract}

Copyright $\odot 2015$ S. Karger AG, Basel

\section{Introduction}

Stroke is the leading cause of adult disability and death worldwide. To reduce the devastating impact of stroke, researchers continuously searched for safe agents which achieve

Q. Yu and Z. Lu contributed equally to this work.

Dr. Xude Sun

and Dr. Qian Ding
Department of Anesthesiology, Tangdu Hospital, Fourth Military Medical University,

Xi'an 710038, (China)

E-Mail sunxude@fmmu.edu.cn and E-Mail qding12345@163.com 
better functional recovery with minimal side-effect in stroke patients. Neuroprotective agents are among one of the most promising approaches to stroke treatment and are applied to protect ischemic neurons in the brain from irreversible injury [1]. One function of neuroprotective agents is to attenuate acute injury to neurons in the ischemic penumbra. Additionally, some of the neuroprotective agents also prevent potentially detrimental events associated with return of blood flow to the brain, termed reperfusion, which may contribute to worse brain injury [2]. During the last two decades, a great deal of efforts have been spent to develop neuroprotective therapies [3]. However, although preclinical studies demonstrated numerous drugs are effective for treating acute stroke in rodents, subsequent clinical trials have been frustrating, and few of the agents have been proven effective.

Hydrogen sulfide $\left(\mathrm{H}_{2} \mathrm{~S}\right)$ has always been considered as a toxic gas and environmental pollutant with an offensive odor. In recent years, emerging evidences indicated $\mathrm{H}_{2} \mathrm{~S}$ exhibited important patho-physiological functions as a novel neural regulatory factor and a potential gaseous neuroprotector in various models from cell culture to human patients [4]. For example, Guo et al. has demonstrated that in $\mathrm{H} 9 \mathrm{c} 2$ cardiac cells, exogenous $\mathrm{H}_{2} \mathrm{~S}$ could attenuate doxorubicin-induced inflammation and cytotoxicity by inhibiting p38 MAPK/NF$\kappa B$ pathway [5]. Recently in a rat model, $\mathrm{H}_{2} \mathrm{~S}$ was reported to protect cardiomyoblasts against oxidative challenge through the inhibition of L-type calcium channels [6]. More importantly in systemic lupus erythematosus patients, $\mathrm{H}_{2} \mathrm{~S}$ was found to inhibit the abnormal activation of lymphocytes through the AKT/GSK3 $\beta$ signal pathway [7]. Moreover, an N-mercapto-based $\mathrm{H}_{2} \mathrm{~S}$ donor was recently found to protect human skin keratinocytes against methylglyoxalinduced injury and behavior dysfunction [8]. Of particular interest to our current study, it has been demonstrated that the administration of $\mathrm{H}_{2} \mathrm{~S}$ significantly ameliorates ischemiareperfusion (I/R) injury in multiple organs. Although the mechanism remained unknown, Kimura et al. reported that $\mathrm{H}_{2} \mathrm{~S}$ improves the glutathione (GSH) levels in the brain under the intrauterine I/R condition [9]. Alternatively, another study demonstrated that $\mathrm{H}_{2} \mathrm{~S}$ effectively benefits neurological function in parallel with a reduction of caspase- 3 in hippocampus and up-regulation of anti-apoptotic protein GSK-3 $\beta$ in cortex in mice with cardiac arrest/ cardiopulmonary resuscitation (CA/CPR) [10]. As an endogenous donor of $\mathrm{H}_{2} \mathrm{~S}, \mathrm{NaHS}$ has been shown to protect against myocardial [11], intestinal [12], hepatic [13] and brain [14] I/R injury. However, the molecular mechanism of its apoptosis inhibition remained controversial.

To investigate the functions of NaHS in I/R induced brain injury, and further elucidate the potential mechanism, we developed the Middle Cerebral Artery Occlusion-reperfusion (MCAO/R) model in rats and examined multiple parameters in the presence or absence of NaHS. Eventually, our results demonstrated that NaHS protected neurons from apoptosis by increasing the activity of superoxide dismutases (SOD) and glutathione peroxidase (GSHPx), which scavenge cellular reactive oxygen species (ROS) and in turn diminish DNA damage and relieve the toxicity from PARP-1 cleavage and AIF translocation. Notably, application of NaHS in MCAO/R also significantly relieved brain injury.

\section{Materials and Methods}

\section{Cells and Animals Treatment}

HT22 cells were purchased from ATCC and maintained in Dulbecco's modified Eagle's medium (Gibicon, USA) plus 10\% fetal bovine serum (Hyclone Laboratories, Logan, UT) and 1\% antibiotics (penicillin/streptomycin). Male Sprague-Dawley rats (220-250 g) were provided by the Animal Center of Fourth Military Medical University. All animals were housed in wire-bottom cages at $25^{\circ} \mathrm{C}$ with a $12 \mathrm{~h}$-light/ dark cycle and fed standard rat chow and water. The care and use of animals in this study followed the guidelines and protocol approved by the Institutional Animal Care and Use Committee (IACUC) of Fourth Military Medical University. All efforts were made to minimize the number of animals used and their suffering. Fourth Military Medical University approved the experimental protocols and governed how the experiments were conducted.

\section{KARGER}




\section{Cellular Physiology Cell Physiol Biochem 2015;36:1539-1551 \begin{tabular}{ll|l} 
and BOI: 10.1159/000430317 & $\begin{array}{l}\text { C 2015 S. Karger AG, Basel } \\
\text { www.karger.com/cpb }\end{array}$ \\
\hline
\end{tabular} \\ Yu et al.: Neuroprotective Effects of NaHS in Brain Injury}

Oxygen-glucose Deprivation and Re-oxygenation (OGD/R)

The HT22 cells were exposed to OGD/R. Briefly, cultured medium was replaced by glucose-free Neurobasal A medium (Life Tech, USA) and the cultured neurons were put in a hypoxic chamber at $37^{\circ} \mathrm{C}$ (Thermo Fisher, USA) with a mix gas containing $5 \% \mathrm{CO}_{2}, 1 \% \mathrm{O}_{2}$ and $94 \% \mathrm{~N}_{2}$ to reach final $2 \% \mathrm{O}_{2}$, which was monitored with $\mathrm{O}_{2}$ analyzer (GODEE, China). After 2 hrs OGD, cells were returned to normal cultured conditions for $48 \mathrm{hrs}$ re-oxygenation.

Cell Viability Assay

Cell viability was measured with CCK-8 (Dojindo Laboratories, Japan) according to the instructions of the manufacturers. In brief, HT22 neurons were seeded in 96-well plates with 50,000 cells/well in DMEM described above. Six wells were prepared for each treatment or control. $10 \mu \mathrm{L}$ of the CCK-8 mixture containing 2-(2-methoxy-4-nitrophenyl)-3-(4-nitrophenyl)-5-(2, 4-disulfophenyl)-2H-tetrazolium, monosodium salt (WST-8) solution was added to each well containing $100 \mu \mathrm{L}$ medium. Cells were incubated at $37^{\circ} \mathrm{C}$ for $3 \mathrm{hrs}$. The ODs were measured at $450 \mathrm{~nm}$ with BioTek's Gen $5^{\mathrm{TM}}$ Microplate Readers (BioTek, Winooski, VT) before and after treatments. Cell viability proportion (CVP) was determined by dividing ODs after the treatments by ODs before the treatment and timed by $100 \%$.

MCAO

The 8-week old rats were initially anesthetized with 3.5\% halothane and maintained with $1 \%$ halothane in $70 \% \mathrm{~N}_{2} \mathrm{O}$ and $30 \% \mathrm{O}_{2}$ by a face mask. Rectal temperature was maintained at $37^{\circ} \mathrm{C}$ throughout the surgical procedure by means of a feedback-regulated water heating system. We induced transient (2 hours) MCAO using a previously described method of intraluminal vascular occlusion [15]. Briefly, a 6-0 thread with a heat-blunted and silicon-coated tip was inserted into the right internal carotid artery through the right external carotid artery. The thread was advanced until it reached the origin of the middle cerebral artery, as evidenced by an abrupt drop in cortical perfusion ( $<25 \%$ of the baseline), which was monitored with a laser Doppler flowmetry (Periflux System 5000, Perimed Inc., Stockholm, Sweden). After 60 min of MCAO, the filament was removed to allow for reperfusion (> 75\% of the baseline). Sham-operated rats underwent the same surgery except for thread insertion. The rats were given with $1.25,2.5$, or $5 \mathrm{mg} / \mathrm{Kg}$ of NaHS intraperitoneally $2 \mathrm{hrs}$ after the onset of MCAO.

\section{2, 3, 5-Triphenyltetrazolium Chloride (TTC) Staining}

2, 3, 5-Triphenyltetrazolium chloride (TTC) Staining were performed by following the protocol described previously [16]. The brains of rats were dissected and sliced using a matrix device (Plastics One Inc., USA) into $2 \mathrm{~mm}$ coronary sections. After medio-sagittal division, one part was stained with 2\% TTC (in saline) for $30 \mathrm{~min}$ at $37^{\circ} \mathrm{C}$ (group I), one part incubated in saline for $30 \mathrm{~min}$ at $37^{\circ} \mathrm{C}$ (group II), and one part was snap frozen (group III). TUNEL staining was carried out using an apoptosis detection kit according to the manufacturer's instructions (Roche Diagnostics GmbH, Germany). Only strongly labeled TUNEL-positive cells were considered as apoptotic, whereas lightly stained cells suggesting necrosis were not evaluated.

\section{Apoptosis Assay}

Apoptosis was examined by the staining with Hoechst33342/PI. Briefly, HT22 cells with or without treatment were incubated in the complete growing medium with Hoechst 33342 and PI at $37^{\circ} \mathrm{C}$ for 30 minutes. Cells were then rinsed twice with PBS and the fluorescent signal was measured with microscope.

\section{Measurement of Oxidative Stress}

Oxidative stress was determined by measuring the activities of superoxide dismutase (SOD) and glutathione peroxidase (GSH-Px), and level of malondialdehyde (MDA). SOD and GSH-Px activities were measured by Superoxide Dismutase and Glutathione Peroxidase Assay Kits (Cayman Chemical, UK) according to the manufacturer's instructions, with enzyme reaction initiated by NADH and terminated by glacial acetic acid, followed by absorbance measured at $560 \mathrm{~nm}$. MDA levels were measured by thiobarbituric acid reactive substances (TBARS) assay according to previously described method [17]. Briefly, $0.1 \mathrm{ml}$ of $10 \%$ homogenates (supplemented with $0.05 \%$ butylated hydroxytoluene) of hippocampus was heated with $0.05 \mathrm{ml}$ thiobarbituric acid for $40 \mathrm{~min}$ at $95^{\circ} \mathrm{C}$. The heated supernatant was re-centrifuged at $3500 \mathrm{rpm}$ for $10 \mathrm{~min}$ and the absorbance was measured at $532 \mathrm{~nm}$. 


\section{Cellular Physiology Cell Physiol Biochem 2015;36:1539-1551 \begin{tabular}{ll|l} 
and Biochemistry 10.1159/000430317 & $\begin{array}{l}\text { P 2015 S. Karger AG, Basel } \\
\text { Published online: July 10, 2015 }\end{array}$ & \begin{tabular}{l} 
www.karger.com/cpb \\
\cline { 2 - 3 }
\end{tabular}
\end{tabular} \\ Yu et al.: Neuroprotective Effects of NaHS in Brain Injury}

\section{Detection of Cellular ATP, ROS and Mitochondrial Membrane Potential}

Cellular ATP levels were determined using a commercially available colorimetric ATP assay kit (Beyotime, China) according to the manufacturer's instructions. ATP levels were normalized to protein levels.

DCFH-DA (Molecular probes, Thermal Fisher, US) was used to measure intercellular ROS level. In brief, HT22 cells with or without OGD treatment were washed twice with pre-warmed serum-free DMEM, followed by incubation with the stain solution ( $5 \mu \mathrm{M}$ DCFH-DA in serum-free medium) at $37^{\circ} \mathrm{C}$ for 20 minutes. Then the fluorescent signal was immediately examined with a fluorescent microscope. The signal intensity was quantified from ten random picked region of interest (ROI) of each cell treatment.

Cellular mitochondrial membrane potential $(\Delta \psi \mathrm{m})$ is measured as described previously [18]. Briefly, $24 \mathrm{hrs}$ after OGD, neurons were pretreated with different concentrations of NaHS for $24 \mathrm{hrs}$ and followed by the incubation with JC-1 (Beyotime). JC-1 forms a monomer at low $\Delta \psi \mathrm{m}$ (green fluorescence; $\lambda$ ex: 490 $\mathrm{nm}$; $\lambda$ em: $530 \mathrm{~nm}$ ) and dimer at higher $\Delta \psi \mathrm{m}$ (red fluorescence; $\lambda$ ex: $520 \mathrm{~nm}$; $\lambda$ em: $590 \mathrm{~nm}$ ). Mitochondrial depolarization was indicated by the ratio of the green/red fluorescence $(\lambda 530 / \lambda 590)$.

$N A D^{+}$Level Assay

Intracellular $\mathrm{NAD}^{+}$levels were measured by using $\mathrm{NAD}^{+} / \mathrm{NADH}$ Assay Kit (Abcam, USA) according to the manufacturer's instructions. Briefly, HT22 cells were washed with cold PBS and extracted with NADH/ NAD extraction buffer by freeze/thaw for two cycles ( $20 \mathrm{~min}$ on ice, then $10 \mathrm{~min}$ at room temperature). Total NAD was detected in a 96-well plate and color was developed and read at $450 \mathrm{~nm}$ by the use of BioTek's $\mathrm{Gen}^{\mathrm{TM}}{ }^{\mathrm{M}}$ Microplate Readers (BioTek) [19].

\section{Subcellular Fractionation}

HT22 cells were cultured to $90 \%$ confluence in $100-\mathrm{mm}$ dishes and subcellular fractionation was performed as described previously [20]. Briefly, cells were washed with ice-cold PBS, then scraped from the dish, and centrifuged (600 g for $5 \mathrm{~min}$ ) to obtain the cell pellet, which was resuspended in lysis buffer (20 mM HEPES-KOH (pH 7.4), $10 \mathrm{mM} \mathrm{NaCl}, 1.5 \mathrm{mM} \mathrm{MgCl}{ }_{2}, 1 \mathrm{mM}$ EDTA, $1 \mathrm{mM}$ EGTA, $250 \mathrm{mM}$ sucrose, and protease inhibitor cocktail). After centrifugation (600 g for $10 \mathrm{~min}$ ), the supernatant was discarded and the pellet was washed and resuspended in nuclear extraction buffer $(20 \mathrm{mM}$ Tris-HCl-pH 7.5, 1.5 $\mathrm{mM} \mathrm{MgCl}, 420 \mathrm{mM} \mathrm{NaCl}, 0.2 \mathrm{mM}$ EDTA, 25\% glycerol, $0.5 \%$ Triton X-100, 0.1\% NP-40, protease inhibitor cocktail), vortexed, and shaken for $30 \mathrm{~min}$. The suspension was then centrifuged (14,000 g for $10 \mathrm{~min})$, and the supernatant containing the nuclear fraction was transferred to a prechilled microcentrifuge tube. All procedures were performed at $4^{\circ} \mathrm{C}$. Total protein was measured using the BCA Protein Assay Kit (Pierce, USA), and the nuclear extracts were used for immunoblotting analysis.

\section{SDS-PAGE and Immunoblotting}

Western blotting was performed accordingly to the methods described previously [21]. Cells or nuclear fractions were lysed in lysis buffer (10 mM HEPES, pH 7.4, 2 mM EGTA, 0.5\% NP-40, protease inhibitors). Equivalent protein quantities $(20 \mu \mathrm{g})$ were subjected to SDS-PAGE, and transferred to nitrocellulose membranes. Membranes were probed with the primary antibodies against PARP-1 (Abcam) and AIF (Abcam), followed by the appropriate HRP-conjugated secondary antibodies (KPL, USA). Immunoreactive bands were visualized with a chemiluminescence kit (Pierce). Intensities of protein bands are quantified by densitometry (ImageJ).

\section{Data Analysis}

In quantitative analyses represented as histograms, values were obtained from three independent experiments, and expressed as the mean \pm SEM. Statistical analysis was performed using the Student's t-test, with $\mathrm{P}$-values $<0.05$ considered significant. ${ }^{*} \mathrm{P}<0.05$ and ${ }^{* *} \mathrm{P}<0.01$ versus the corresponding controls are indicated. All statistical data were calculated with GraphPad Prism software (GraphPad Software, USA). 


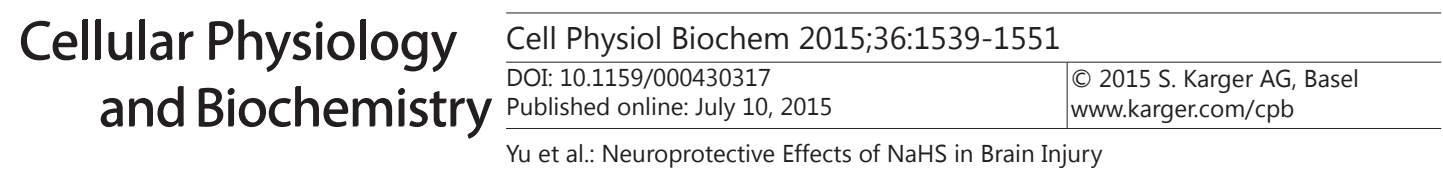

\section{Results}

NaHS protects brain from ischemia/reperfusion-induced damage

Hydrogen sulfide $\left(\mathrm{H}_{2} \mathrm{~S}\right)$ is an essential signal molecule in the brain. Pretreatment of rats with the $\mathrm{H}_{2} \mathrm{~S}$ releasing agent, NaHS, improves focal cerebral ischemia/reperfusion (I/R) injury [14]. We first titrated NaHS dosage in HT22 cells and did not find any toxicity with different concentrations of NaHS from 0 to $250 \mu \mathrm{M}$ (Fig. 1A). Consistent with previous study, sub-lethal dosage of NaHS treatment significantly protected HT22 cells from oxygen-glucose deprivation (OGD) induced cell death (Fig. 1A and B). To further examine the effect of NaHS in vivo, the $\mathrm{MCAO} / \mathrm{R}$ model was established in rats. Of note, increased NaHS concentration is correlated with the reduced modified neurological severity scores (mNSS), the most commonly used neurological scale in animal studies of stroke. The results indicated the capability of NaHS treatment to improve the circulation in stroke (Fig. 1C). Consistent with in vitro observations, TTC staining showed that the brain infarct from NaHS treated rats was significantly reduced in a dosage-dependent manner (Fig. 1D and E). These findings indicated a potent neuroprotective effect of NaHS.

NaHS improves brain injury by preventing OGD/MCAO-induced neuronal apoptosis

Large amounts of free radicals are generated during cerebral ischemia/reperfusion, and oxidative stress plays an important role in brain damage after stroke. In addition to oxidizing macromolecules that lead to cell damage, oxidative stresses are also involved in apoptosis signaling pathways and cause mitochondrial dysfunction. Experimental data from laboratory animals that either overexpress or are deficient in antioxidant proteins,

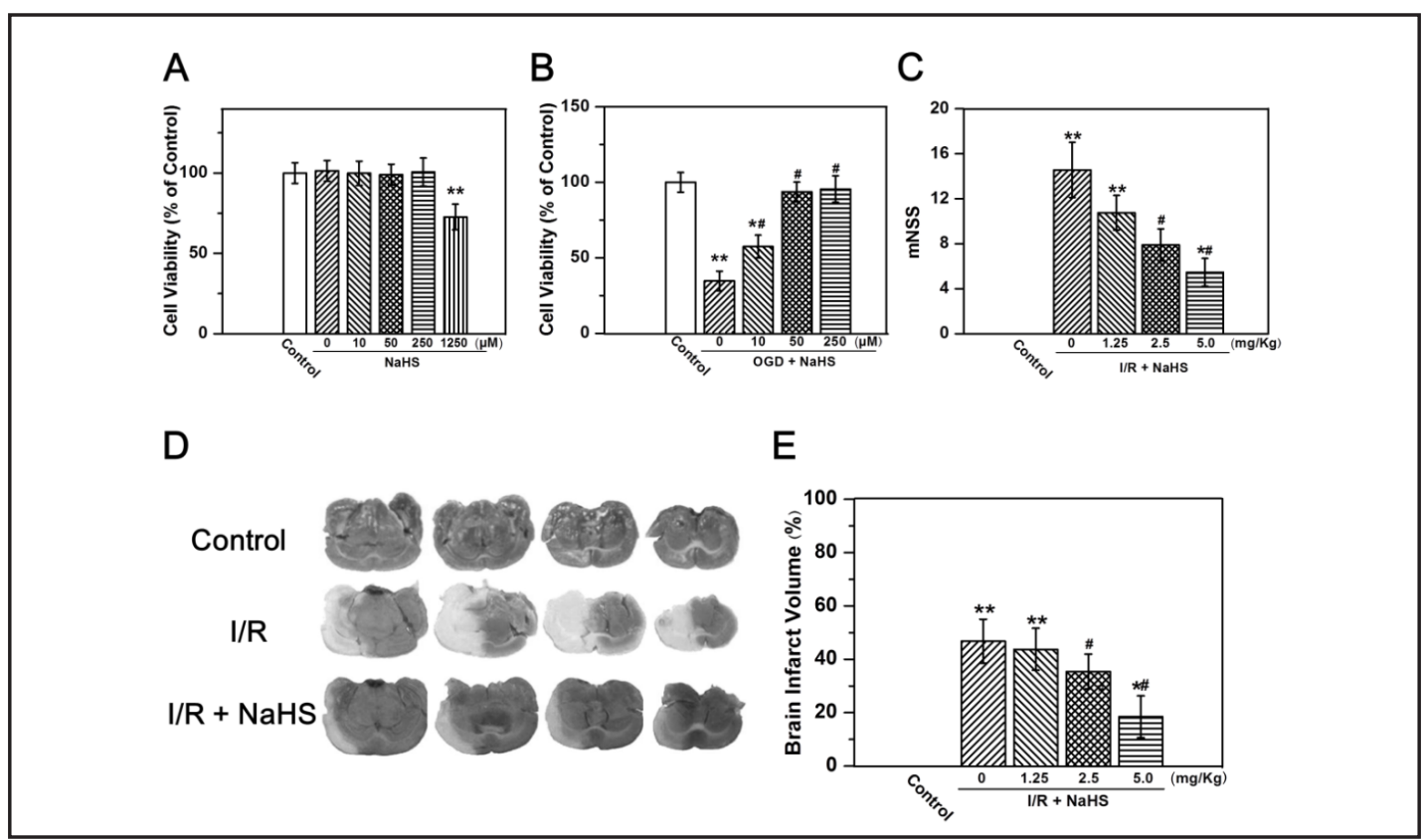

Fig. 1. Neuroprotective effect of NaHS in OGD and MCAO models. (A) Histogram showing the cell viability of HT22 cells exposed to different concentrations of NaHS for $24 \mathrm{~h}$, as measured by CCK-8 assay. ${ }^{* *} P<0.01$ versus control. (B) Histogram showing the cell viability of control cells, HT22 cells were exposed to OGD and then treated with 10,50 , or $250 \mu \mathrm{M}$ of NaHS, as measured by CCK- 8 assay. ${ }^{*} P<0.05,{ }^{* *} P<0.01$ versus control, $\# P<0.05$ versus OGD alone (control). (D) Representative TTC staining images of rat brain slices in control, I/R and I/R plus NaHS $(2.5 \mathrm{mg} / \mathrm{Kg})$ administration. Histograms respectively showing quantitative comparison of the mNNS (C) and the brain infarct volume (E) for sham-operated rats (control), or for rats subjected to MCAO (I/R) and then administered with $1.25,2.5$, or $5.0 \mathrm{mg} / \mathrm{Kg}$ of NaHS. The data were represented as the mean \pm SEM from three independent experiments. ${ }^{*} P<0.05$, ${ }^{* *} P<0.01$ versus control, $\# P<0.05$ versus $0 G D$ alone. 
Fig. 2. Inhibition of OGD-induced cellular apoptosis in HT22 cells by NaSH. (A) Representative fluorescence micrographs of Hoechst-PI staining for HT22 cells cultured in the plain medium (control), exposed to OGD alone, exposed to OGD and then treated with 10 , 50 , or $250 \mu \mathrm{M}$ of NaHS. Scale bar indicates $20 \mu \mathrm{m}$. The resulting histogram (B) showing the percentage of apoptotic cells (PI-positive) in cell population after different cell treatments

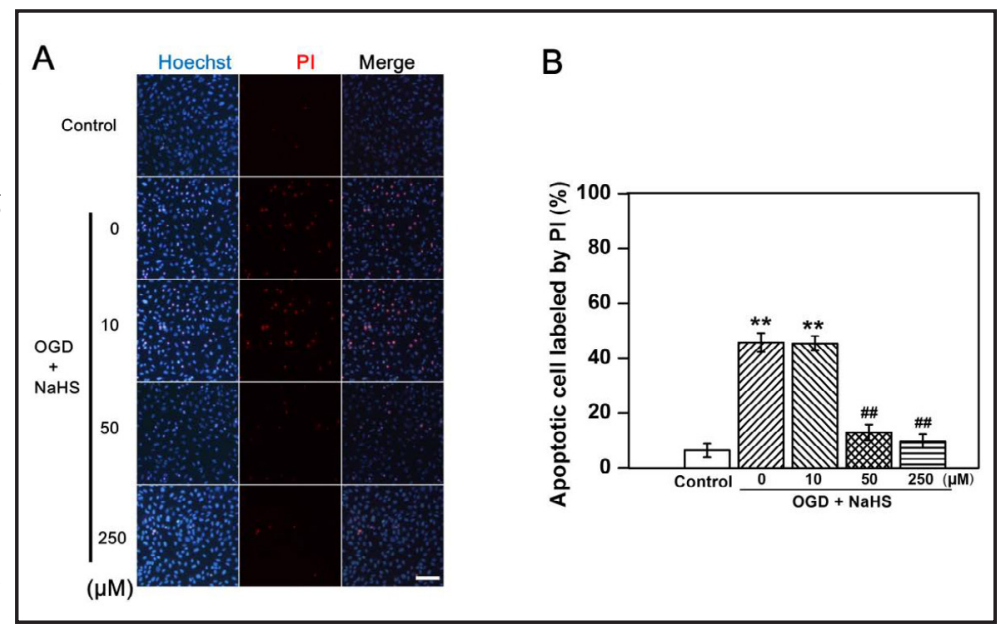
as indicated. The data were represented as the mean \pm SEM from three independent experiments. ${ }^{*} P<0.05$, $* * P<0.01$ versus Control, $\# P<0.05$, \#\#P<0.01 versus OGD alone.

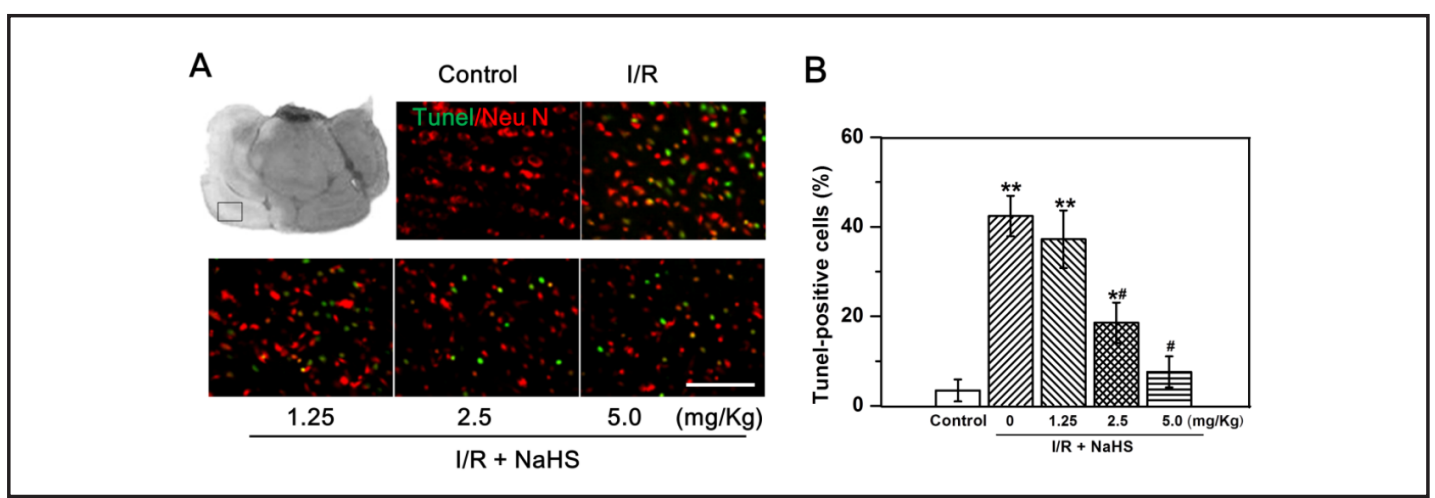

Fig. 3. Inhibition of MCAO-induced neuronal apoptosis in rat brain by NaSH. Representative fluorescence micrographs of TUNEL staining for the cerebral cortex (A) of sham-operated rats (control), or for rats subjected to MCAO (I/R) and then administered with 1.25, 2.5, or $5 \mathrm{mg} / \mathrm{Kg}$ of NaHS. Scale bar indicates $20 \mu \mathrm{m}$. The resulting histogram (B) showing the percentage of apoptotic cells (TUNEL-positive) in cell population in the cerebral cortex for rats as indicated above. The data were represented as the mean \pm SEM from three independent experiments. ${ }^{*} P<0.05,{ }^{* *} P<0.01$ versus control, $\# P<0.05$ versus I/R alone.

mainly superoxide dismutase family members, have provided strong evidences on the role of oxidative stress in neuronal apoptosis and ischemic brain damage [22]. To our knowledge, both OGD and MCAO triggers brain injury via the induction of apoptosis in neurons. It is therefore intriguing to address whether NaHS protects neurons from ischemic stress by preventing apoptosis. HT22 cells under OGD condition exhibited significantly enhanced cell death, as indicated by the percentage of cells with positive staining of propidium iodide (PI) compared with untreated cells (6.2\% vs $42.9 \%)$. Notably, in those cells permeable to PI, hoechst33342 staining showed clear chromatin condensation, which is the hallmark of apoptosis (Fig. 2A). Furthermore, when combined with NaHS treatment, OGD-induced apoptosis was largely inhibited $(42.9 \%$ vs $12.8 \%$ in the presence of $50 \mu \mathrm{M}$ NaHS and $9.7 \%$ in the presence of $250 \mu \mathrm{M}$ NaHS), suggesting NaHS indeed protected neuron cells from apoptosis (Fig. 2B). Consistently, NaHS treatment inhibited neuronal apoptosis, as indicated by TUNEL assay in the cerebral cortex of rat subjected to SHAM or MCAO treatment (Fig. 3A and B). Collectively, NaHS plays a plausible role in protecting ischemia-induced brain injury by preventing neuronal apoptosis.

\section{KARGER}


Fig. 4. Inhibition of OGD-induced ROS production by NaSH. (A) Representative fluorescence micrographs of DCFH-DA staining for HT22 cells cultured in the plain medium (control), exposed to OGD alone, exposed to OGD and then treated with 10,50 , or $250 \mu \mathrm{M}$ of NaHS. Scale bar indicates $20 \mu \mathrm{m}$. The intracellular ROS levels were measured by a fluorescence microscopy using the fluorescent probe DCFH-DA. (B) Quantification of fluorescent intensity was detected at an excitation wavelength of $488 \mathrm{~nm}$. The data were represented as the mean \pm SEM from three independent experiments. ${ }^{*} P<0.05,{ }^{* *} P<0.01$ versus Control, \#P<0.05 versus OGD alone.

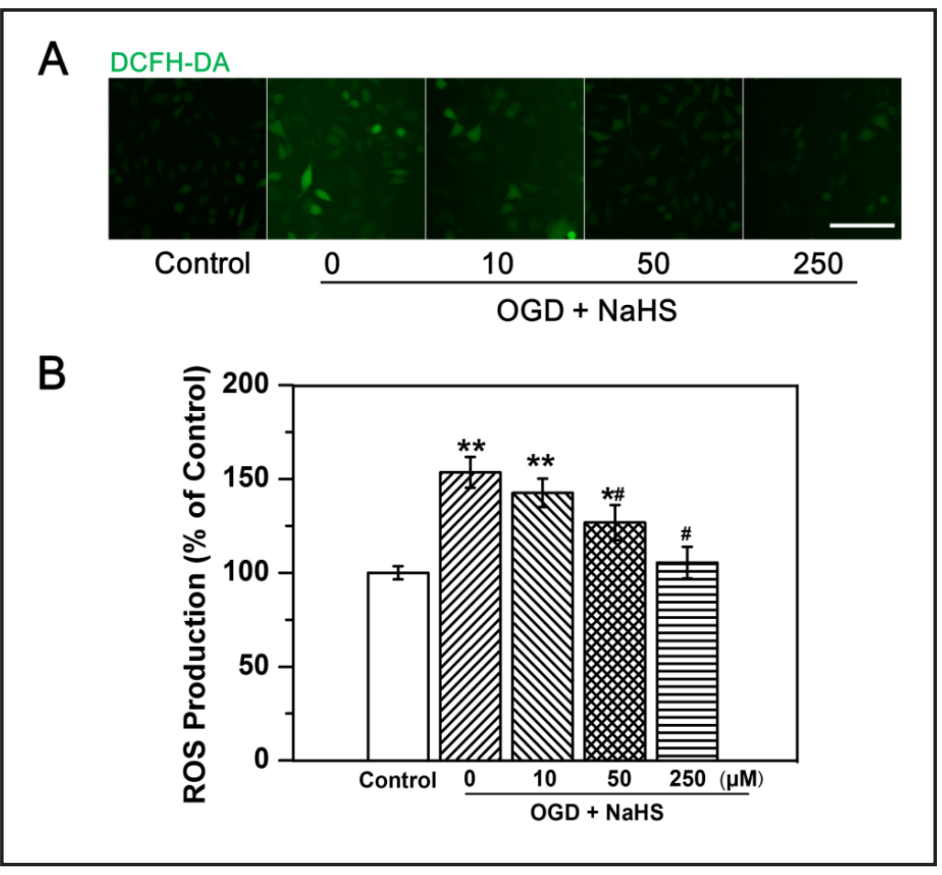

NaHS inhibits neuronal apoptosis by reducing reactive oxygen species (ROS)

Mitochondria play a critical role in reperfusion injury by producing excessive reactive oxygen species (ROS) that damage cellular components, and initiating cell death [23]. Interestingly, $\mathrm{H}_{2} \mathrm{~S}$ has been demonstrated to protect cells from apoptosis by removing cellular ROS [24], which prompted us to examine the ROS status in the presence or absence of NaHS. HT22 cells were pretreated with OGD and followed by treatment with increasing dosage of NaHS. Cellular ROS was then measured by a cell-permeable ROS probe, DCFH-DA. As expected, NaHS reduced ROS level in a dosage-dependent manner (Fig. 4A and 4B), which was consistent with its inhibitory effect in OGD-induced neuronal apoptosis and the protective function in MCAO-induced brain injury. To understand how NaHS neutralized cellular ROS, we then examined the effect of NaHS on antioxidant pools and malondialdehyde (MDA) in rat brain. As shown in Fig. 5, ischemia/reperfusion treatment reduced the activity of both superoxide dismutases (Cu/Zn SOD) (Fig. 5A) and glutathione peroxidase (GSH-Px) (Fig. $5 \mathrm{~B}$ ), but increased MDA contents, which is one of the terminal products of lipid peroxidation and a hallmark of ROS (Fig. 5C). This effect was however significantly attenuated by NaHS treatment, suggesting that NaHS inhibited neuronal apoptosis by enhancing SOD and GSHPx, which neutralized cellular ROS.

\section{NaHS improves OGD-induced mitochondrial dysfunction}

Mitochondria are the powerhouse of cells. The primary function of mitochondria is to generate adenosine triphosphate (ATP) through oxidative phosphorylation via the electron transport chain. During ATP production, the leakage of electrons to oxygen results in the generation of ROS, which serve as signal messenger molecules at the physiological level. However, accumulation of ROS may cause genome instability and subsequently activate cellular safeguard machinery, such as p53. The signal was then transduced back to mitochondria, causing increased mitochondrial outer membrane permeabilization (MOMP) and release of pro-apoptotic factors from inter-membrane space, thereby triggering the apoptosis cascades [25].

Mitochondrial dysfunction has been identified as a key pathological event, and oxidative stress plays an important role in brain damage after stroke [26]. The precise molecular changes that occur within mitochondria of brain during I/R injury remain unclear. Among the 

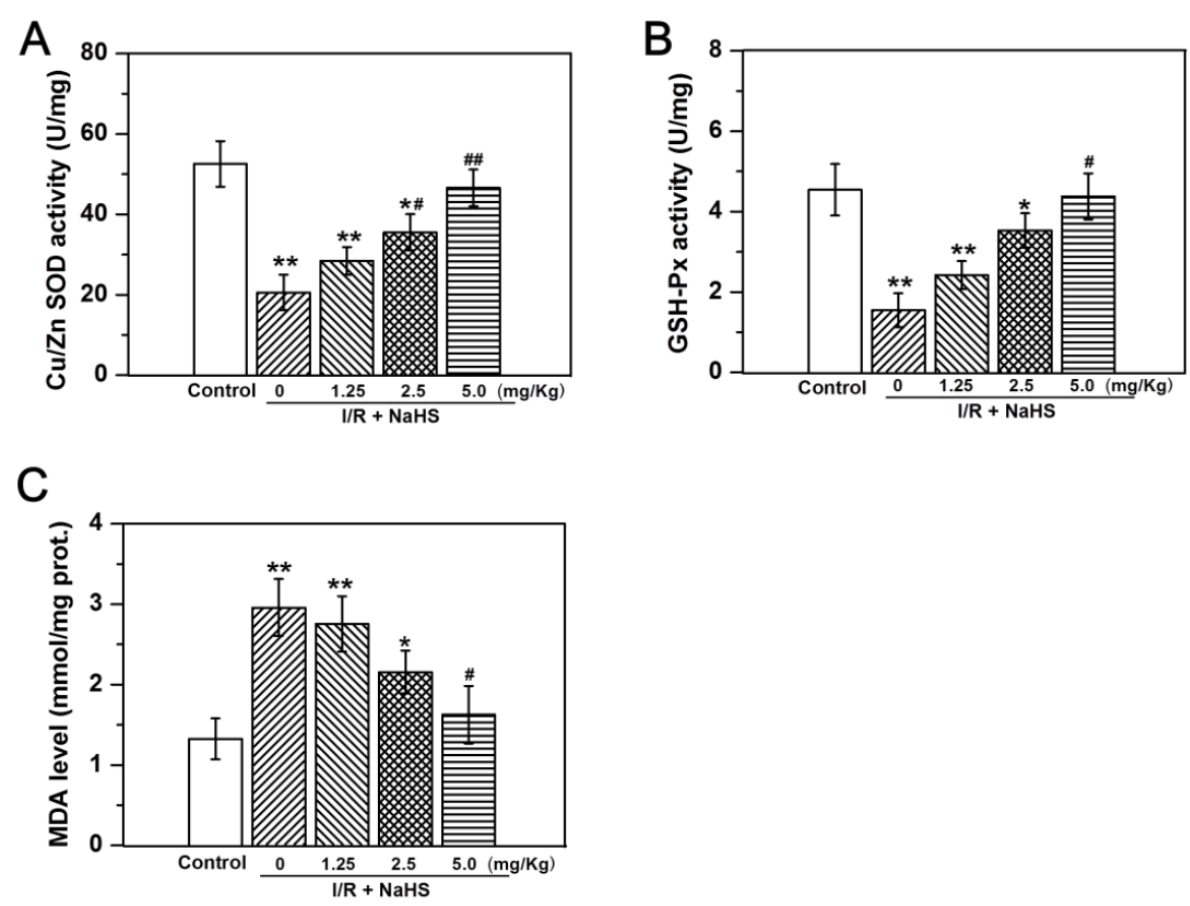

Fig. 5. NaSH amelioration of oxidative stress after I/R injury. NaSH with different concentrations significantly enhanced superoxide dismutase (SOD) (A) and glutathione peroxidase (GSH-Px) (B) and decreased the content of malondialdehyde (MDA) (C) activities in I/R rats. Data were expressed as mean \pm SEM $(n=8)$. ${ }^{*} P<0.05,{ }^{* *} P<0.01$ versus control, $\# P<0.05$, \#\#P<0.01 versus I $\mathrm{R}$ alone.
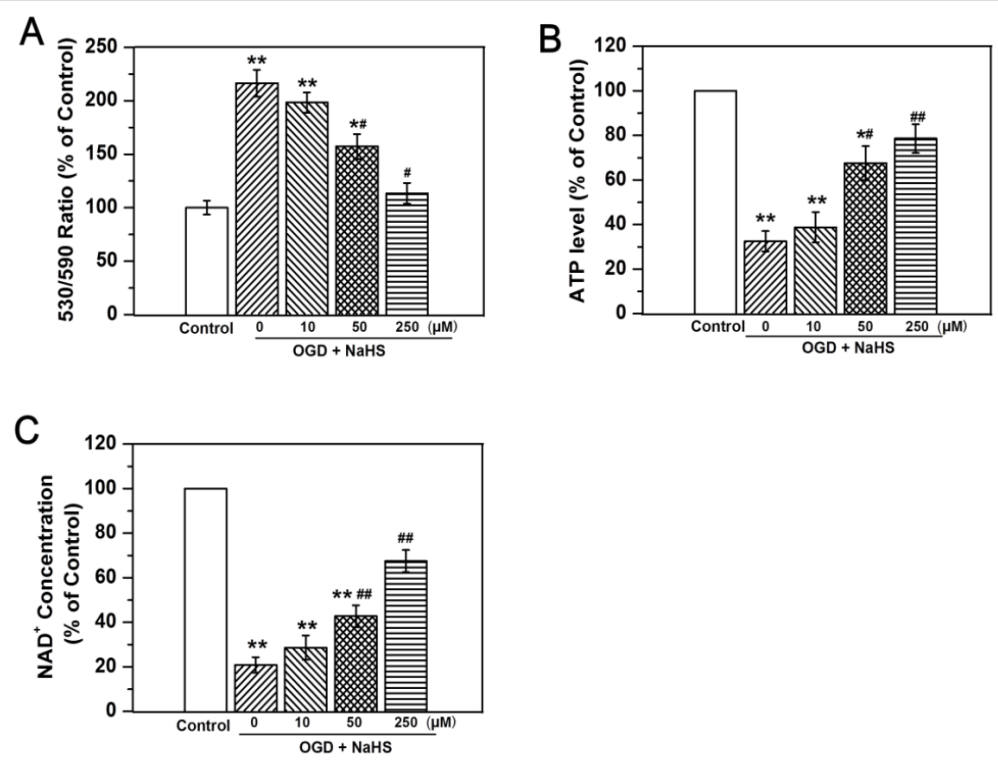

Fig. 6. Inhibition of OGD-induced mitochondrial dysfunction by NaSH. $\Delta \psi \mathrm{m}(\mathrm{A}), \mathrm{ATP}(\mathrm{B})$, and NAD ${ }^{+}(\mathrm{C})$ were assayed. The data were represented as the mean \pm SEM from three independent experiments. ${ }^{*} P<0.05$, ${ }^{* *} P<0.01$ versus Control, $\# P<0.05$, \#\#P<0.01 versus 0 GD alone. The data were represented as the mean \pm SEM from three independent experiments. ${ }^{*} P<0.05$, ${ }^{* *} P<0.01$ versus Control, $\# P<0.05$ versus OGD alone.

few characterized mechanisms in cardiac ischemia-reperfusion model, it has been reported that mitochondrial respiratory complexes I, III, IV and V, and many enzymes involved 

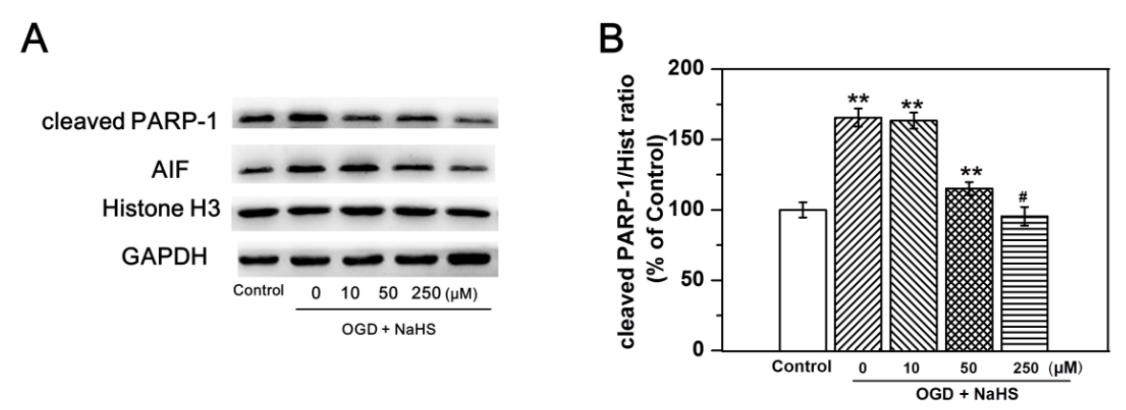

C

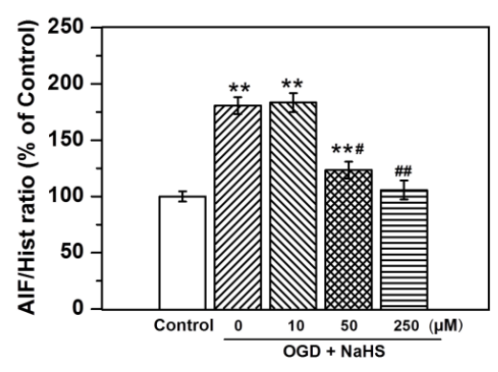

Fig. 7. NaHS inhibition of OGD-induced nuclear translocation of PARP-1 and AIF. (A) Western blot analysis of PARP-1, AIF, Histone H3 nuclear protein and GAPDH protein of HT22 cells cultured in the plain medium (Control), exposed to OGD alone, exposed to OGD and then treated with 10, 50, or $250 \mu \mathrm{M}$ of NaHS for 24 h. (B-C) Relative optical densities of PARP-1, AIF bands. Data were expressed as mean \pm SEM. $* P<0.05$, ${ }^{* *} P<0.01$ versus control, $\# P<0.05$, \#\#P<0.01 versus 0 GD alone.

in Krebs cycle are all affected by I/R injury [22, 27-29]. These defects in the respiratory machinery directly cause mitochondrial dysfunction, which is characterized by reduced oxygen consumption and impaired ATP production. Moreover, $\mathrm{H}_{2} \mathrm{~S}$ was found to be oxidized to thiosulfate through the sequential action of sulfide quinone oxidoreductase (SQR) and rhodanese in the mitochondria [30]. Therefore, it is possible that NaHS exerts its protective function from oxidative stress by releasing $\mathrm{H}_{2} \mathrm{~S}$ in the mitochondria hence restoring its function. As the driving force of protons into mitochondrial matrix through ATP synthase, mitochondrial membrane potential $(\Delta \psi \mathrm{m})$ is commonly used to evaluate the integrity of mitochondrial inner membrane. Loss of $\Delta \psi \mathrm{m}$ is correlated with decreased ATP production and the slowdown of electron transport, which is characterized by the reduction of $\mathrm{NAD}^{+} /$ NADH ratio. Similar to previous report [19], OGD treatment reduces $\Delta \psi \mathrm{m}$ (Fig. 6A), ATP production (Fig. 6B) as well as $\mathrm{NAD}^{+}$(Figure $6 \mathrm{C}$ ) in HT22 cells, recapitulating oxidative stressinduced mitochondrial dysfunction. Notably, the addition of NaHS led to dosage-dependent improvement of the deficiency in mitochondrial structural and functional integrity (Fig. 6 A-C).

NaHS inhibits oxidative stress-induced apoptosis through the inhibition of AIF nuclear translocation and PARP-1 cleavage

$\mathrm{NaHS}$, as an exogenous donor of $\mathrm{H}_{2} \mathrm{~S}$, has been shown to protect against myocardinal [11], intestinal [12], hepatic [13] and brain [14] I/R injury. However, the molecular mechanism of its apoptosis inhibition remained controversial. Zhang and his colleague demonstrated that both nuclear translocation of the apoptosis-inducing factor (AIF) and cleavage of poly (ADP-ribose) polymerase-1 (PARP-1) contribute to I/R-induced cerebral endothelial cell apoptosis [20]. To investigate whether NaHS protects brain I/R injury through similar mechanism, HT22 cells were treated with OGD followed by either immunoblotting or nuclear fractionation analyses. In Fig. 7A and 7B, OGD-induced PARP-1 cleavage was largely inhibited 
by the addition of NaHS. Other than the cleavage of PARP-1, OGD treatment also resulted in AIF nuclear translocation (Fig. 7C), and NaHS treatment consistently reduced AIF levels in the nuclear fractions. These findings suggested that NaHS inhibited neuronal apoptosis with the involvement of AIF and PARP-1 inhibition.

\section{Discussion}

In this study, we presented experimental evidences for the first time dissecting the underlying mechanism responsible for the observed NaHS protective effect on I/R injury. We found that NaHS inhibits AIF nuclear translocation and PARP-1 cleavage (Fig. 7), both of which were reported to contribute to I/R-induced cerebral endothelial cell apoptosis. We speculate that inhibition of these processes by NaHS could rescue apoptotic events caused by increased cellular ROS level.

To protect the brain from additional injury during reperfusion, various strategies are employed to discover and develop neuroprotective agents, such as preventing white blood cells from adhering to vessel walls, limiting formation of free radicals and promoting neuronal repair which attenuates neuronal apoptosis [3]. Increasing evidences suggested $\mathrm{H}_{2} \mathrm{~S}$ is a novel type of endogenous neural regulatory factor and gaseous mediator [4]. In particular, it has been demonstrated that the administration of $\mathrm{H}_{2} \mathrm{~S}$ significantly ameliorates ischemia-reperfusion (I/R) injury in multiple organs. As the $\mathrm{H}_{2} \mathrm{~S}^{2}$ releasing agent, NaHS has been shown to reduce the adhesion of white blood cells, hyperplasia and hypertrophy of cardiac fibroblast, and improve left ventricular systolic function and diastolic function, thereby largely decrease the mortality rate of rats after myocardial I/R injury [11]. Our current result clearly proved that NaHS played a robust role in protecting neurons from cell death, indicating potential therapeutic function of NaHS in stroke treatment.

Our results showed that the protective effect of NaHS is well correlated with reduction of ROS and increased SOD and GSH-Px activities, which indicates that the beneficial outcome of NaHS may be secondary to the scavenging of ROS by $\mathrm{H}_{2} \mathrm{~S}$ or secondary to the up-regulation of antioxidants. SOD catalyzes the conversion of superoxide radicals to hydrogen peroxide, therefore alleviates the toxic effects of ROS. We showed that treatment with NaHS significantly increased the activity of cerebral SOD. The significantly higher levels of SOD promoted by NaHS should contribute to its protective effect by diminishing superoxide and neutralizing hydrogen peroxides and lipoperoxides, which were consistently demonstrated in Fig. 4 and Fig. 5. Apparently NaHS inhibits the focal I/R injury, at least partially by enhancing the activity of antioxidant SOD, which in turn scavenges the I/R-related ROS, mitigating the focal cerebral I/R injury in rats. Meanwhile, we have shown that the activity of GSH-Px was significantly impaired by focal cerebral I/R, whereas administration of NaHS restored GSHPx activity in the presence of focal cerebral I/R treatment. Reduced glutathione (GSH) is one of the critical detoxification systems of cellular ROS and is postulated to act as a firstline defense against myocardial I/R injury [31]. Together with glutathione reductase, which catalyzes the conversion of oxidized GSH back to its reduced form, GSH-Px is important in regulating the glutathione cycle. Previous studies have demonstrated an important role of cellular GSH-Px in protection of the myocardium from I/R injury. Our results suggested that replenish of GSH-Px activity may represent another important mechanism underlying NaHS protection against oxidative damage during focal cerebral I/R injury.

PARP-1 is a chromatin-associated zinc finger protein responsible for catalyzing the transfer of the ADP-ribose moiety from its substrate, the oxidized form of nicotinamide adenine dinucleotide $\left(\mathrm{NAD}^{+}\right)$, to diverse nuclear acceptor proteins [32,33]. PARP-1 is activated by the DNA strand breaks, and the extensive activation can initiate an energy-consuming futile intracellular cycle, leading to rapid depletion of cellular stores of $\mathrm{NAD}^{+}$and ATP, which results in cell dysfunction and cell death [33]. Cell-based studies demonstrated that diverse apoptotic stimuli, including oxidative stress with concomitant energy depletion, serum deprivation and alkylating agents, cause a caspase-mediated cleavage of the PARP-1, thereby

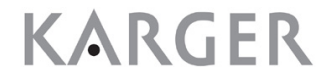


allowing the cell to preserve ATP levels required for energy-dependent apoptosis and to release the suppression of apoptosis by poly(ADP-ribosyl)ated histone H1 [34]. Additionally, in vivo studies showed that inactivation of the PARP-1 gene in mice lead to pronounced protection in cerebral and myocardial ischemia-reperfusion injury $[35,36]$ and hemorrhagic shock [37]. AIF is a pro-apoptotic flavoprotein which normally functions as an oxidoreductase and mitochondrial antioxidant. In response to apoptotic stimuli, AIF translocates from the mitochondria to the nucleus, where it triggers chromatin condensation and apoptosis [38]. Interestingly, AIF may be a key downstream effector of PARP-1-mediated cell death. Studies in cultured neurons and fibroblasts indicate that PARP-1 activation induced by DNA damage results in the poly(ADP-ribosyl)ation of nuclear proteins, which subsequently translocate to the mitochondria to trigger the translocation of AIF from the intermembrane space to the nucleus to cause chromatin condensation and DNA fragmentation [39]. In our study, we found that NaHS treatment clearly inhibited PARP-1 cleavage and AIF translocation, which is consistent with the neuronal protective effect of NaHS.

\section{Conclusion}

Taken together, we hereby demonstrated that NaHS treatment exhibited significant effect in antagonizing neuronal apoptosis and improved the focal cerebral I/R-induced brain damage. Our findings shed new light on the discovery of neuroprotective agents that could potentially contribute to the therapy of stroke. Nonetheless, the potential of $\mathrm{H}_{2} \mathrm{~S}$ in the relief of brain injury has been shown to be concentration-sensitive. Higher dose of $\mathrm{H}_{2} \mathrm{~S}$ have been shown to magnify cerebral damage [40] and administration of high concentration of NaHS significantly increased cerebral infarct volume in rats following MACO [41]. Therefore, although our results strongly suggested that administration of $\mathrm{NaHS}$ has potential therapeutic function in clinical treatment of stroke, translating its pharmacological effects represents a big challenge and further studies are needed to address the function of NaHS and the downstream mechanisms.

\section{Acknowledgements}

This study was supported by the National Natural Science Foundation of China (81201024, 81271343).

\section{Disclosure Statement}

The authors declare that they have no conflict of interest.

\section{References}

1 Green AR: Protecting the brain: the search for a clinically effective neuroprotective drug for stroke. Crit Rev Neurobiol 2004;16:91-97.

2 Hallenbeck JM, Dutka AJ: Background review and current concepts of reperfusion injury. Arch Neurol 1990;47:1245-1254.

3 Cheng YD, Al-Khoury L, Zivin JA: Neuroprotection for ischemic stroke: two decades of success and failure. NeuroRx 2004;1:36-45.

4 Lowicka E, Beltowski J: Hydrogen sulfide (H2S) - the third gas of interest for pharmacologists. Pharmacol Rep 2007;59:4-24. 


\section{Cellular Physiology Cell Physiol Biochem 2015;36:1539-1551 \begin{tabular}{l|l|l}
\hline DOI: 10.1159/000430317 & C 2015 S. Karger AG, Basel
\end{tabular}

5 Guo R, Wu K, Chen J, Mo L, Hua X, Zheng D, Chen P, Chen G, Xu W, Feng J: Exogenous hydrogen sulfide protects against doxorubicin-induced inflammation and cytotoxicity by inhibiting p38MAPK/NFkB pathway in H9c2 cardiac cells. Cell Physiol Biochem 2013;32:1668-1680.

6 Avanzato D, Merlino A, Porrera S, Wang R, Munaron L, Mancardi D: Role of calcium channels in the protective effect of hydrogen sulfide in rat cardiomyoblasts. Cell Physiol Biochem 2014;33:1205-1214.

7 Han Y, Zeng F, Tan G, Yang C, Tang H, Luo Y, Feng J, Xiong H, Guo Q: Hydrogen sulfide inhibits abnormal proliferation of lymphocytes via AKT/GSK3 $\beta$ signal pathway in systemic lupus erythematosus patients. Cell Physiol Biochem 2013;31:795-804.

8 Yang CT, Zhao Y, Xian M, Li JH, Dong Q, Bai HB, Xu JD, Zhang MF: A novel controllable hydrogen sulfidereleasing molecule protects human skin keratinocytes against methylglyoxal-induced injury and dysfunction. Cell Physiol Biochem 2014;34:1304-1317.

9 Kimura Y, Goto Y, Kimura H: Hydrogen sulfide increases glutathione production and suppresses oxidative stress in mitochondria. Antioxid Redox Signal 2010;12:1-13.

10 Minamishima S, Bougaki M, Sips PY, Yu JD, Minamishima YA, Elrod JW, Lefer DJ, Bloch KD, Ichinose F: Hydrogen sulfide improves survival after cardiac arrest and cardiopulmonary resuscitation via a nitric oxide synthase 3-dependent mechanism in mice. Circulation 2009;120:888-896.

11 Johansen D, Ytrehus K, Baxter GF: Exogenous hydrogen sulfide (H2S) protects against regional myocardial ischemia-reperfusion injury--Evidence for a role of K ATP channels. Basic Res Cardiol 2006;101:53-60.

12 Liu H, Bai XB, Shi S, Cao YX: Hydrogen sulfide protects from intestinal ischaemia-reperfusion injury in rats. J Pharm Pharmacol 2009;61:207-212.

13 Kang K, Zhao M, Jiang H, Tan G, Pan S, Sun X: Role of hydrogen sulfide in hepatic ischemia-reperfusioninduced injury in rats. Liver Transpl 2009;15:1306-1314.

14 Yin J, Tu C, Zhao J, Ou DM, Chen GW, Liu Y, Xiao XZ: Exogenous hydrogen sulfide protects against global cerebral ischemia/reperfusion injury via its anti-oxidative, anti-inflammatory and anti-apoptotic effects in rats. Brain Research 2013;1491:188-196.

15 Chen J, Li Y, Wang L, Zhang Z, Lu D, Lu M, Chopp M: Therapeutic benefit of intravenous administration of bone marrow stromal cells after cerebral ischemia in rats. Stroke 2001;32:1005-1011.

16 Kramer M, Dang J, Baertling F, Denecke B, Clarner T, Kirsch C, Beyer C, Kipp M: TTC staining of damaged brain areas after MCA occlusion in the rat does not constrict quantitative gene and protein analyses. J Neurosci Methods 2010;187:84-89.

17 Buege JA, Aust SD: Microsomal lipid peroxidation. Methods Enzymol 1978;52:302-310.

18 Gao XY, Huang JO, Hu YF, Gu Y, Zhu SZ, Huang KB, Chen JY, Pan SY: Combination of mild hypothermia with neuroprotectants has greater neuroprotective effects during oxygen-glucose deprivation and reoxygenation-mediated neuronal injury. Sci Rep 2014;4:7091.

19 Xu H, Luo P, Zhao Y, Zhao M, Yang Y, Chen T, Huo K, Han H, Fei Z: Iduna protects HT22 cells from hydrogen peroxide-induced oxidative stress through interfering poly(ADP-ribose) polymerase-1-induced cell death (parthanatos). Cell Signal 2013;25:1018-1026.

20 Zhang Y, Zhang X, Park TS, Gidday JM: Cerebral endothelial cell apoptosis after ischemia-reperfusion: role of PARP activation and AIF translocation. J Cereb Blood Flow Metab 2005;25:868-877.

21 Liu Y, Cheng H, Zhou Y, Zhu Y, Bian R, Chen Y, Li C, Ma Q, Zheng Q, Zhang Y, Jin H, Wang X, Chen Q, Zhu D: Myostatin induces mitochondrial metabolic alteration and typical apoptosis in cancer cells. Cell Death Dis 2013;4:e494.

22 Chen H, Yoshioka H, Kim GS, Jung JE, Okami N, Sakata H, Maier CM, Narasimhan P, Goeders CE, Chan PH: Oxidative stress in ischemic brain damage: mechanisms of cell death and potential molecular targets for neuroprotection. Antioxid Redox Signal 2011;14:1505-1517.

23 Sanderson TH, Reynolds CA, Kumar R, Przyklenk K, Huttemann M: Molecular mechanisms of ischemiareperfusion injury in brain: pivotal role of the mitochondrial membrane potential in reactive oxygen species generation. Mol Neurobiol 2013;47:9-23.

24 Shen Y, Guo W, Wang Z, Zhang Y, Zhong L, Zhu Y: Protective effects of hydrogen sulfide in hypoxic human umbilical vein endothelial cells: a possible mitochondria-dependent pathway. Int J Mol Sci 2013;14:1309313108.

25 Niizuma K, Endo H, Chan PH: Oxidative stress and mitochondrial dysfunction as determinants of ischemic neuronal death and survival. J Neurochem 2009;109:133-138. 


\section{Cellular Physiology Cell Physiol Biochem 2015;36:1539-1551 \begin{tabular}{ll|l} 
and Biochemistry & $\begin{array}{l}\text { DOI: 10.1159/000430317 } \\
\text { Published online: July 10, } 2015\end{array}$ & $\begin{array}{l}\text { O) 2015 S. Karger AG, Basel } \\
\text { www.karger.com/cpb }\end{array}$ \\
\cline { 2 - 3 } & &
\end{tabular} \\ Yu et al.: Neuroprotective Effects of NaHS in Brain Injury}

26 Lesnefsky EJ, Moghaddas S, Tandler B, Kerner J, Hoppel CL: Mitochondrial dysfunction in cardiac disease: ischemia--reperfusion, aging, and heart failure. J Mol Cell Cardiol 2001;33:1065-1089.

27 Rouslin W: Mitochondrial complexes I, II, III, IV, and V in myocardial ischemia and autolysis. Am J Physiol 1983;244:H743-748.

28 Lesnefsky EJ, Tandler B, Ye J, Slabe TJ, Turkaly J, Hoppel CL: Myocardial ischemia decreases oxidative phosphorylation through cytochrome oxidase in subsarcolemmal mitochondria. Am J Physiol 1997;273:H1544-1554.

29 Lucas DT, Szweda LI: Declines in mitochondrial respiration during cardiac reperfusion: age-dependent inactivation of alpha-ketoglutarate dehydrogenase. Proc Natl Acad Sci U S A 1999;96:6689-6693.

30 Kimura H: Hydrogen sulfide and polysulfides as biological mediators. Molecules 2014;19:16146-16157.

31 Haramaki N, Stewart DB, Aggarwal S, Ikeda H, Reznick AZ, Packer L: Networking antioxidants in the isolated rat heart are selectively depleted by ischemia-reperfusion. Free Radic Biol Med 1998;25:329-339.

32 Ikejima M, Noguchi S, Yamashita R, Ogura T, Sugimura T, Gill DM, Miwa M: The zinc fingers of human poly(ADP-ribose) polymerase are differentially required for the recognition of DNA breaks and nicks and the consequent enzyme activation. Other structures recognize intact DNA. J Biol Chem 1990;265:2190721913.

33 Ha HC, Snyder SH: Poly(ADP-ribose) polymerase-1 in the nervous system. Neurobiol Dis 2000;7:225-239.

34 Yoon YS, Kim JW, Kang KW, Kim YS, Choi KH, Joe CO: Poly(ADP-ribosyl)ation of histone H1 correlates with internucleosomal DNA fragmentation during apoptosis. J Biol Chem 1996;271:9129-9134.

35 Endres M, Wang ZQ, Namura S, Waeber C, Moskowitz MA: Ischemic brain injury is mediated by the activation of poly(ADP-ribose)polymerase. J Cereb Blood Flow Metab 1997;17:1143-1151.

36 Pieper AA, Walles T, Wei G, Clements EE, Verma A, Snyder SH, Zweier JL: Myocardial postischemic injury is reduced by polyADPripose polymerase-1 gene disruption. Mol Med 2000;6:271-282.

37 Liaudet L, Soriano FG, Szabo E, Virag L, Mabley JG, Salzman AL, Szabo C: Protection against hemorrhagic shock in mice genetically deficient in poly(ADP-ribose)polymerase. Proc Natl Acad Sci U S A 2000;97:10203-10208.

38 Hansen TM, Nagley P: AIF: a multifunctional cog in the life and death machine. Sci STKE 2003;2003:PE31.

39 Hong SJ, Dawson TM, Dawson VL: Nuclear and mitochondrial conversations in cell death: PARP-1 and AIF signaling. Trends Pharmacol Sci 2004;25:259-264.

40 Boysen G, Brander T, Christensen H, Gideon R, Truelsen T: Homocysteine and risk of recurrent stroke. Stroke 2003;34:1258-1261.

41 Qu K, Chen CP, Halliwell B, Moore PK, Wong PT: Hydrogen sulfide is a mediator of cerebral ischemic damage. Stroke 2006;37:889-893. 